\title{
Simple laboratory set-up for the fringe-projection method
}

Ibnur Muhd, Gerald Liew, Mark Wee, Theresa Lai, Yu Fu, et al.

Ibnur Rashad B. Zainal A. Muhd, Gerald M. Liew, Mark Wee, Theresa Lai, Yu Fu, Huai Min Shang, "Simple laboratory set-up for the fringe-projection method," Proc. SPIE 4588, Seventh International Conference on Education and Training in Optics and Photonics, (28 May 2002); doi: 10.1117/12.468718

Event: Education and Training in Optics and Photonics 2001, 2001, Singapore, Singapore 


\title{
A Simple Laboratory Set-up For The Fringe-Projection Method
}

\author{
Muhd Ibnur Rashad B. Zainal A. ${ }^{\text {a }}$, Gerald Liew Ming Jie ${ }^{a}$, Mark Wee ${ }^{a}$, Theresa Lai ${ }^{a}$, \\ Y. Fu ${ }^{b}$, H.M. Shang ${ }^{b^{*}}$ \\ ${ }^{a}$ Raffles Institution, 1 Raffles Institution Lane, Singapore 575954 \\ ${ }^{b}$ Department of Mechanical Engineering, National University of Singapore, 10 Kent Ridge Crescent, \\ Singapore 119260 \\ *Email: mpeshm@nus.edu.sg
}

\begin{abstract}
In recent years, optical fringe-projection and other optical interferometric techniques for surface profiling have received much attention because they are whole-field and non-contacting; very high data processing speeds can be achieved using computer image-processing techniques. These advantages over many other mechanical probe-based techniques are particularly useful for the measurement of large surfaces as well as for micro-systems at the sub-micron level.

In the fringe-projection technique, a reference optical grating is first generated and then projected onto the surface of interest. For a given optical set-up, the distribution of the reference grating is perturbed in accordance with the profile of the test surface, thereby enabling direct derivation of surface profiles from measurements of the perturbed fringe distribution. The reference gratings are readily generated with a Michelson interferometer, which uses a beam-splitting cube and mirrors - these optical elements are readily available in all laboratories. A major drawback of this technique is the need for good vibration isolation, as otherwise unstable fringes will be generated. Alternatively, beam-splitting cubes with coated reflective surfaces can be used, but this would not allow adjustment of the frequency of the generated fringes.

This paper describes a very simple method of generating and projecting optical grating for surface profiling. The working principle is based on the reflection-refraction of a commercial beam-splitting cube. By carefully adjusting the orientation of the laser beam, the frequency of the grating can be varied. A distinct advantage of this method over the Michelson interferometer lies in its ability to generate stable carrier fringes under lax vibration isolation conditions.
\end{abstract}

Keywords: interferometry, Young's fringes, fringe projection, profile measurement

\section{INTRODUCTION}

Common optical methods for surface profiling include the use of moiré and holographic interferometry, fringe projection and others. ${ }^{1-13}$ Among them, the fringe projection method is perceived as the simplest one because optical fringes can be generated and projected easily with a Michelson interferometer or a LCD projector. The use of the Michelson interferometer requires stringent vibration-free testing conditions, thereby confining its applications to laboratory environment. The use of a LCD projector does not require stringent vibration isolation but this involves high capital investment. This paper presents a very simple method for generating and projecting optical gratings onto a test surface. The working principle is based on the reflection-refraction of a commercially available beam-splitting cube. By varying the angle of incidence of the laser beam, the frequency of the optical grating that is generated can be easily adjusted. Furthermore, this method does not require the use of many optical elements; therefore the need for vibration isolation can be lax.

\section{GENERATION AND PROJECTION OF OPTICAL FRINGES}

As shown in Figure 1, a laser beam with incidence angle $\theta$ is refracted at Point $E$ and later split at Point $O$ on the hypotenuse of a beam-splitting cube. The two beams $O A C$ and $O B D$ exiting from the beam-splitting cube are expanded using a converging lens that is placed in front of the cube. Linear Young's fringes are generated by the interference of the 
exit beams $A C$ and $B D$, and they are subsequently projected onto the test surface that is placed at distance $\mathrm{L}$ from the converging lens. When the test surface is a flat screen and when $L$ is much larger than the distance $h$ between $C$ and $D$, the pitch $p$ of the generated fringes is given by the following expression.

$p=\frac{L \lambda}{h}$

where $L$ is the distance between the converging lens and flat screen, $\lambda$ is the wavelength of the laser source, and $h$ is the distance between $C$ and $D$.

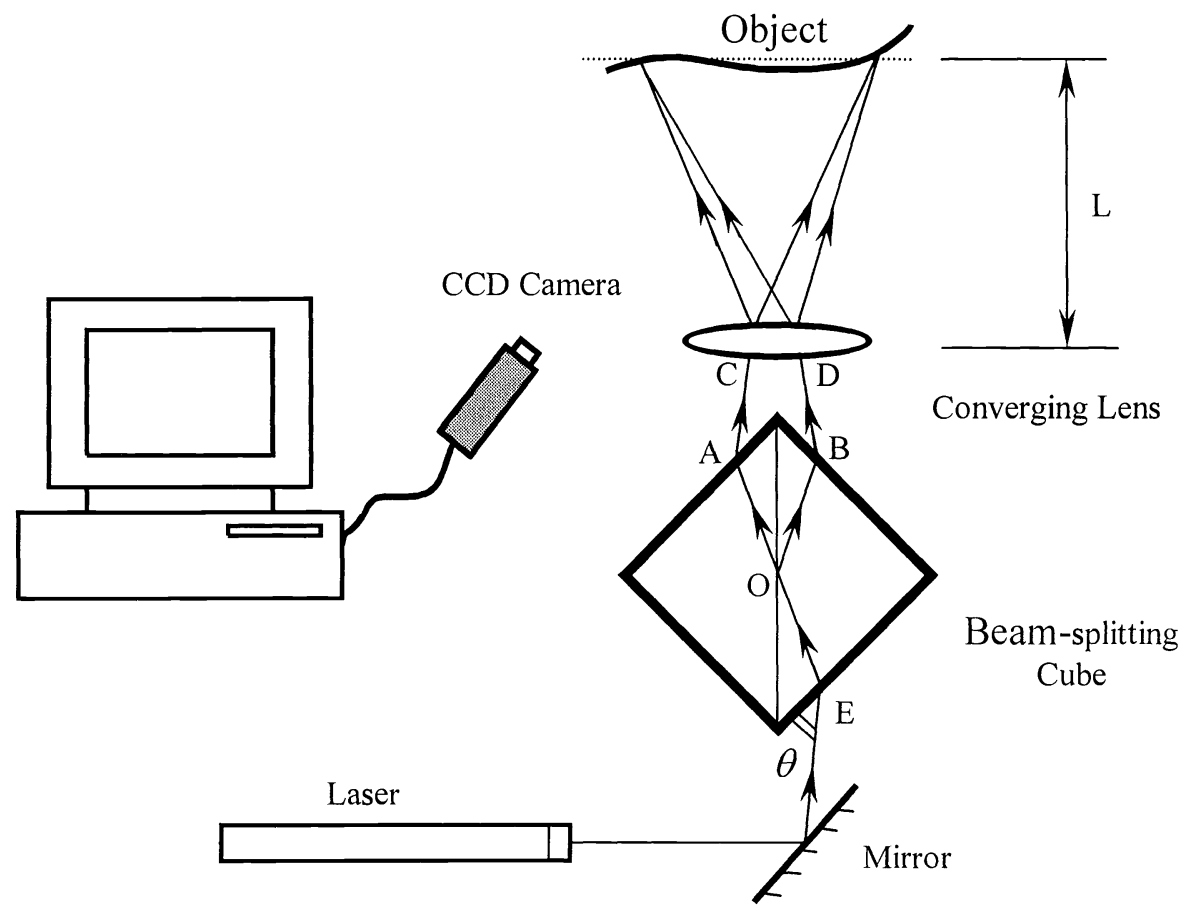

Fig. 1. Generation and projection of optical linear fringes

The distance $h$ between $C$ and $D$ can be varied by carefully adjusting the incidence angle $\theta$, resulting in a change in the pitch of the grating. When the flat screen is replaced by a test surface, the distribution of the grating is perturbed in accordance with the profile of the test surface. The CCD camera shown in Figure 1 captures the image of the perturbed fringe pattern for subsequent analysis using a computer system. The fringe pattern is subsequently analyzed using the Fast Fourier Transform method together with a suitable phase unwrapping algorithm. ${ }^{14,15}$

\section{EXPERIMENTAL ILLUSTRATION}

Figure 2 shows the perturbed fringe pattern that is observed on the surface of a 3-D object - a paperweight (50mm by $70 \mathrm{~mm}$ ) resembling the face of a lion. Figures 3 to 5 show respectively the wrapped phase map, the unwrapped phase map, and the reconstructed surface profile using Fast Fourier Transform.

The optical set-up illustrated in Figure 1 may also be used for profiling the surface of a large object. Figure 6 shows the fringe pattern that is observed on a cover $0.8 \mathrm{~m}$-width and $1 \mathrm{~m}$-height. Figure 7 shows a 3 -D plot of the surface. 


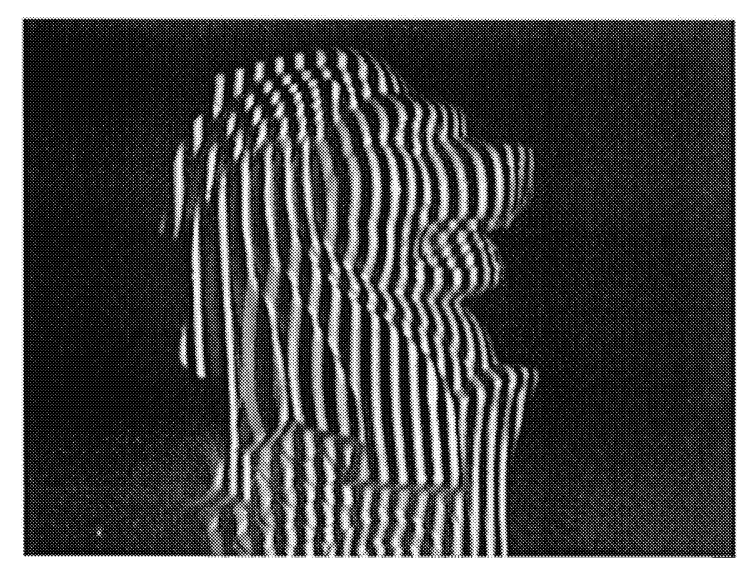

Fig. 2. Perturbed fringe patterns on a paperweight

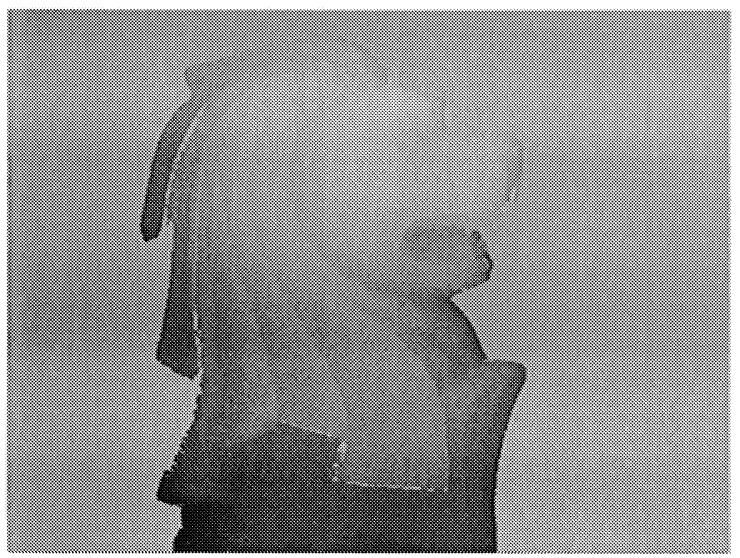

Fig. 4. Gray scale map of the paperweight

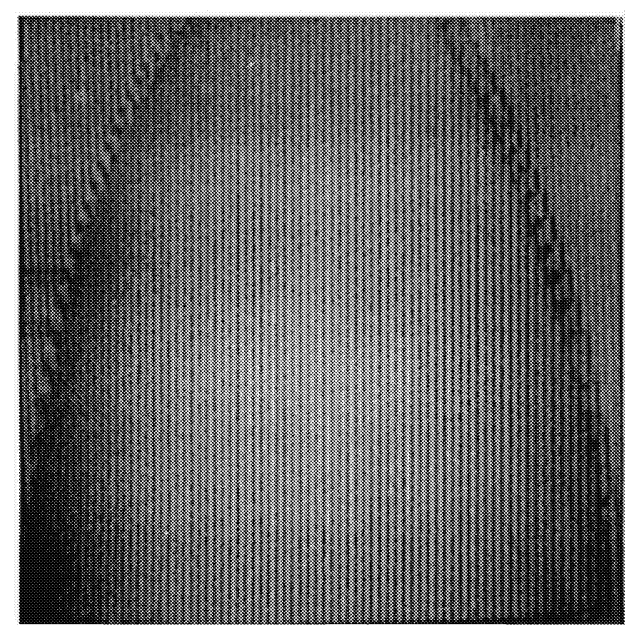

Fig. 6. Purturbed fringe patterns on a cover

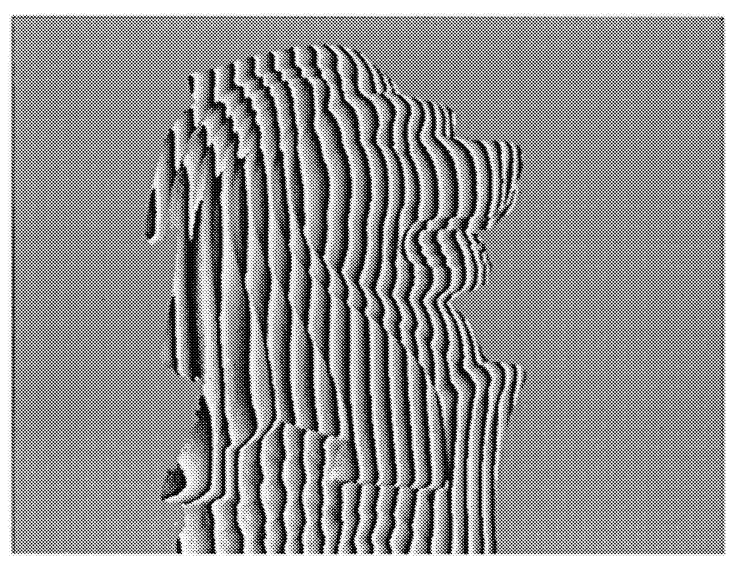

Fig. 3. Wrapped phase map of the paperweight

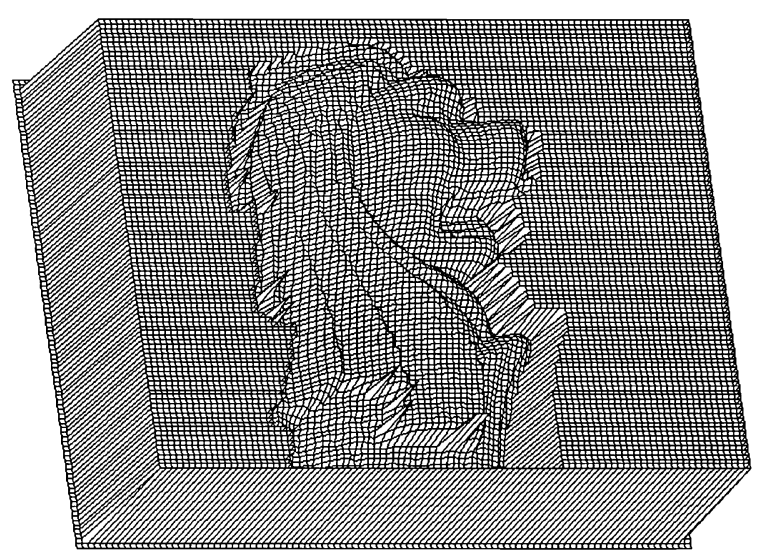

Fig. 5. A 3-D plot of the paperweight

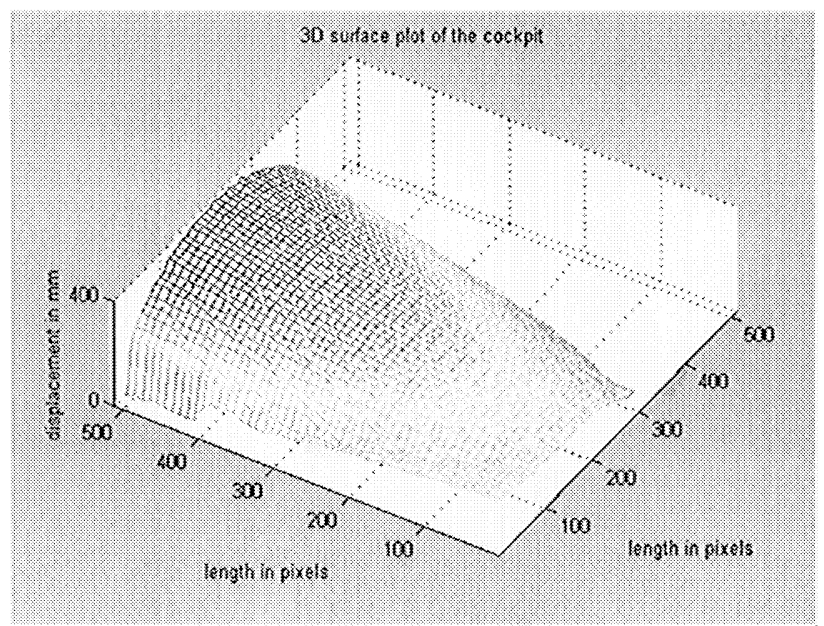

Fig. 7. A 3D-plot of the cover 


\section{CONCLUDING REMARKS}

In this paper, a fringe-projection method for three-dimensional surface contouring using a commercial beam-splitting cube is described. A distinct advantage of this method over the Michelson interferometer lies in its ability to generate stable fringes with lax vibration isolation. Furthermore, the density of the fringes can be varied by changing the incidence angle of the laser beam. This subsequently enables the measurement of both large and small components.

\section{REFERENCES}

1. T. Yatagai, M. Idesawa, "Automatic fringe analysis for moiré topography", Opt Laser Eng, 3, 73-83 (1982).

2. Y.Z. Dai, F P.Chiang, "Contouring by moiré interferometry" Exp Mech, 31, 76-81(1991).

3. J. D. Hovanesian, Y. Y. Hung, "Moire contour-sum contour difference, and vibration analysis of arbitrary objects", Appl Opt, 10, 2734 (1971).

4. R. Thalmann, R. Dandliker, "Holographic contouring using electronic phase measurement", Opt Eng, 24(6), 930-935 (1985).

5. C. Quan, H. M. Shang, C. J. Tay and P. J. Bryanston-Cross, "Holographic contouring using double-source technique and Fourier transform analysis", Opt Laser Eng, 30, 351-362 (1998).

6. P. K. Rastogi, "Visualisation of the contours of equal slope of an arbitrarily-shaped object using holographic moiré", Opt Eng, 33(7), 2373-2377 (1994).

7. M. Takeda, K. Mutoh, "Fourier transform profilometry of 3-D diffuse objects by spatial phase detection", Appl Opt, 25(10), 1630-33(1986).

8. M. Suganuma, T. Yoshizawa, "Three-dimensional shape analysis by use of a projected grating image", Opt Eng, 30 (10), 1529-1533(1991).

9. M. K. Kalm, W. Juptner and W. Osten, "Automatic adaption of projected fringe pattern using a programmable LCD-projector", Proc SPIE, 3100, 156-165 (1997).

10. M. Halioua, H.C.Liu, "Optical three-dimensional sensing by phase measuring profilometry", Opt Laser Eng, 11(3), 185-215(1989).

11. X. He, D. Zou, S. Liu and Y. Guo, "Phase-shifting analysis is moiré interferometry and its applications in electronic packaging", Opt Eng, 37(5), 1410-1419 (1998).

12. P. S. Huang, Q. Hu, F. Jin and F. P. Chiang, "Color-encoded fringe projection and phase shifting for 3-D surface contouring", Proc SPIE, 3407, 477-482(1998).

13. X. Y. Su, W. S.Zhou, "Automated phase-measuring profilometry using defocused projection of a Ronchi grating", Opt. Communi, 94, 561(1992).

14. K. Creath, "Phase-measurement interferometry techniques", Prog. In Opt, 26, 349-393(1988).

15. C. Quan, X.Y.He, C. J. Tay, H. M. Shang, "3D surface profile measurement using LCD fringe projection". Proc SPIE, 4317, 511-516 (2000). 